Bio-grafia: Escritos sobre la Biología y su Enseñanza Vol 3 No5 ISSN 2027-1034. Segundo semestre de 2010, Bogotá, Colombia, pp 64-82

\title{
LAS PRÁCTICAS DE CAMPO EN LA CONSTRUCCIÓN DEL CONOCIMIENTO PROFESIONAL DE FUTUROS PROFESORES DE BIOLOGÍA
}

\section{${ }^{1}$ THE FIELD PRACTICUMS IN THE CONSTRUCTION OF} PROFESSIONAL KNOWLEDGE OF FUTURE BIOLOGY TEACHERS.

Por: Amórtegui Elías1; Gutiérrez Alice Marcela2; Medellín Francisco.3.

\begin{tabular}{|l|}
\hline Recibido: $19-09-2010$ \\
\hline Aceptado: 06-12-2010 \\
\hline
\end{tabular}

\section{RESUMEN}

El presente escrito muestra el trabajo de práctica pedagógica I y II desarrollado al interior del Grupo de Investigación Conocimiento Profesional del Profesor de Ciencias, en la Universidad Pedagógica Nacional (UPN) durante el 2007-2008 con estudiantes de sexto semestre de Licenciatura en Biología, con el fin de contribuir a la construcción del Conocimiento Profesional del Profesor de Biología, a través de las prácticas de campo como estrategia en la enseñanza de la biología.

En un primer acercamiento, se indagó las concepciones de los estudiantes acerca de la relación entre las prácticas de campo y el Conocimiento Profesional del Profesor de Biología, evidenciando que consideraban la importancia de éstas, únicamente, a partir de su rol como aprendices y su incidencia en la construcción del Conocimiento Biológico, sin tener en cuenta la importancia de éstas en su quehacer como docentes. En este sentido, como contenido formativo favorecer en los estudiantes de sexto semestre, la práctica de campo como una estrategia en la enseñanza de la biología, para esto se plantearon talleres, reflexiones y un espacio en el cual los futuros profesores diseñaron una práctica de campo para alumnos de segundo grado de educación básica de una Institución Educativa Distrital.

Dichos espacios y reflexiones permitieron a los futuros maestros, considerar la práctica de campo como una estrategia en la enseñanza de la

1. ${ }^{1}$ Estudiante de Décimo semestre de Licenciatura en Biología de la Universidad Pedagógica Nacional. Tesista y Monitor de Investigación del Grupo Conocimiento Profesional del Profesor de Ciencias. folkerpan@hotmail.com.

2.Profesora Departamento de Biología, Universidad Pedagógica Nacional. galicemarcela@hotmail.com.

3.Profesor Departamento de Biología, Universidad Pedagógica Nacional. franciscomedellin@hotmail.com 
biología la cual aporta a la construcción de un Conocimiento propio del profesor de biología.

PALABRAS CLAVE: Conocimiento Profesional del Profesor de Biología (CPPB), Conocimiento Biológico (CB), Conocimiento Didáctico del Contenido Biológico (CDCB), Prácticas de Campo, Enseñanza de la Biología.

\section{ABSTRACT}

This document shows the work of pedagogical practicum I and II developed within the research group Conocimiento Profesional del Profesor de Biologia, in the Universidad Pedagógica Nacional (UPN) with students of sixth semester of Biology teaching degreeer, with the purpose of contributing to the construction of the Professional Knowledge of Biology Teacher through the field work as a strategy in Biology teaching.

In a first approach the students conceptions about the relationship between the field practicums and the professional knowledge of the Biology teacher were investigated,finding out that the importance of field praticums is considered only from

their role as students and their effect on the construction of biological knowledge without taking into account their importance for their teaching task. In that sense, it is important like a learning content to favor the pedagogical practicum as a strategy in Biology teaching in the sixth semester students, by means of workshops, reflections and a space in which the future teachers designed a field practicum for students of the second grade of secondary in a public educative institution.

Such spaces and reflections allowed the future teaches to consider the pedagogical practicum as a strategy in Biology teaching, which contributes to the construction of own knowledge about the Biology teacher.

KEYWORDS: Professional Knowledge of The Biology Teacher, Biological Knowledge, Didactic Knowledge Of The Biological Content, Field Work, Biology Teaching.

\section{INTRODUCCIÓN}

El profesor debe contar con un Conocimiento particular acerca de la metodología, las estrategias y actividades de enseñanza de tópicos específicos de la ciencia, lo cual implica conocer modelos, ejemplos, analogías, ilustraciones, problemas, demostraciones, simulaciones, entre otros. Lo anterior no desde la perspectiva instrumental, sino que es menester el Conocimiento acerca de cada actividad, es decir, saber hasta qué punto se potencia el establecimiento de interrelaciones, en qué medida facilita la superación de obstáculos, entre otros. Se requiere entonces, realizar transformaciones del Conocimiento Cotidiano, del Conocimiento Científico, principalmente, para facilitar la comprensión por parte de los alumnos de los 
principales conceptos y la construcción de un Conocimiento escolar.

El CDCB le permite al profesor realizar la transposición didáctica del Conocimiento Biológico, lo cual implica la definición, organización, secuenciación, desarrollo, y regulación de los contenidos escolares y de las estrategias metodológicas de enseñanza de la Biología en el aula (incluida la evaluación), de tal manera que se pueda facilitar y favorecer el aprendizaje por parte de los alumnos (Valbuena 2007).

Según Del Carmen y Pedrinaci (1997), dentro de dichas estrategias de enseñanza, es fundamental el uso del entorno y del trabajo de campo. Es importante que el docente trabaje con detenimiento en el diseño de las salidas de campo tanto en la organización como en sus objetivos educativos, de tal manera que a partir de esos objetivos formativos claros que pretende el maestro en su formación, proyecte el tipo de salida que quiere diseñar, preguntándose ¿A dónde ir?, ¿Cuándo ir?, el grado de conocimiento del lugar, el tiempo disponible, y así adecuar los objetivos y contenidos, la posibilidad de que el alumno trabaje con cierta autonomía; y así plantear una actividad de iniciación, una actividad de reestructuración, una actividad de síntesis y una actividad evaluativa. También se debe tener en cuenta los principales tipos de salidas, su organización, su equipamiento. Es importante además que los estudiantes en formación participen en el proceso de preparación y desarrollo de las salidas de campo para que luego puedan diseñarlas para una futura aplicación.

La relación entre la formación del profesorado y los trabajos prácticos ha venido constituyendo una faceta muy valorada entre los enseñantes (Boyer y Tiberghien 1989; Rodrigo 1993; García Barros 1998; citados en Rodrigo et al 1999). Los estudios realizados sobre la efectividad de estas actividades, son escasos, al igual que los estudios específicos de concepciones del profesorado relacionados con maneras de abordar el trabajo práctico. Para este estudio es esencial conocer las ideas de partida del futuro profesorado y así hacer una aproximación a las concepciones que tienen sobre el trabajo práctico. Las labores de investigación e indagación sobre las prácticas que realiza el profesorado y cómo pueden mejorar, se presentan como una necesidad evidente en el panorama actual de la Didáctica de las Ciencias (Rodrigo et al, 1999); de aquí la pertinencia e importancia de este trabajo, que permitió identificar en una primera medida las ideas de los formadores (refiriéndose a los profesores de los futuros profesores) y de los estudiantes (refiriéndose a los futuros docentes) sobre las prácticas de campo, permitiendo entonces diseñar y generar espacios para posibilitar que dichas ideas pudieran progresar con respecto a la importancia de las prácticas de campo en la enseñanza de la biología y en la formación inicial de profesores. 
Este trabajo se enmarca en un enfoque cualitativo, en donde la investigación es de tipo exploratoria buscando dar significado social, encontrando resultados ideográficos e interpretativos, bajo este enfoque la realidad social debe ser construida junto con los miembros de la sociedad (Bonilla y Rodríguez, 1997); En cuanto al método, se circunscribe en el método hermenéutico de Miles y Huberman (1994), el cual busca interpretar y dar significado a una realidad investigada. De acuerdo a lo anterior, se hizo uso de herramientas como encuestas con el fin de contextualizar el grupo de trabajo, entrevistas para indagar las ideas de los maestros con relación a las Prácticas de Campo a partir de la modificación de LDI: The Laboratory Dimensions Inventory citado en Del Carmen, 2000 (Ver Figura 1); y cuestionarios a partir de la modificación de la propuesta de Rodrigo et al, 1999 (Ver anexo 1) para indagar las ideas de los futuros maestros con relación a las prácticas y su incidencia en el CPPB, además de actividades como talleres, discusiones y el espacio para que los futuros maestros diseñaran una Práctica de Campo para segundo grado de educación básica.

\begin{tabular}{|l|l|}
\hline CATEGORIA & CARACTERISTICAS \\
\hline $\begin{array}{l}\text { DIMENSIÓN SOCIAL } \\
\text { (Forma) }\end{array}$ & $\begin{array}{l}\text { ¿Cómo se organizan los estudiantes para trabajar durante } \\
\text { la práctica?, ¿A dónde y cuántas son las prácticas?, ¿Cuáles } \\
\text { son los objetivos de las prácticas?, ¿Cómo es el } \\
\text { acompañamiento de los profesores? }\end{array}$ \\
\hline TIPO DE SALIDA & $\begin{array}{l}\text { ¿Qué enfoque se maneja en las prácticas de campo?, ¿Cómo } \\
\text { es la evaluación de la práctica de campo? }\end{array}$ \\
\hline $\begin{array}{l}\text { CONOCIMIENTOS } \\
\text { PREVIOS (Integración) }\end{array}$ & $\begin{array}{l}\text { ¿Con que componente/s se relaciona la práctica de campo?, } \\
\text { ¿Los estudiantes conocen la teoría cuando realizan la } \\
\text { práctica? }\end{array}$ \\
\hline $\begin{array}{l}\text { RELACIÓN TEORÍA- } \\
\text { PRACTICA }\end{array}$ & $\begin{array}{l}\text { ¿La teoría es básica para desarrollar la práctica?, ¿De la } \\
\text { teoría a la práctica o de la práctica a la teoría?, ¿La práctica } \\
\text { esta pensada para enseñar un concepto clave?, ¿Se ayuda a } \\
\text { que el estudiante supere las ideas previas y se aproximen al } \\
\text { trabajo científico? }\end{array}$ \\
\hline $\begin{array}{l}\text { OBTENCIÓN DE LOS } \\
\text { DATOS }\end{array}$ & $\begin{array}{l}\text { ¿Cómo obtienen los datos los estudiantes durante la } \\
\text { práctica? }\end{array}$ \\
\hline $\begin{array}{l}\text { ANÁLISIS DE LOS } \\
\text { DATOS }\end{array}$ & $\begin{array}{l}\text { ¿Qué tipo de análisis de pide a los estudiantes? ¿Existe } \\
\text { alguna orientación sobre la mejor manera de expresar, } \\
\text { presentar y comunicar los datos? }\end{array}$ \\
\hline $\begin{array}{l}\text { ¿Cómo Se está aportando a la formación profesional a } \\
\text { FORMACIÓN } \\
\text { PROFESIONAL del trabajo con las prácticas de campo? }\end{array}$ \\
\hline
\end{tabular}

Figura 1: Categorías de entrevista a los coordinadores de semestres. Modificado de The Laboratory Dimensions Inventory según Tamir y García 1992, citados en Del Carmen (2000). 
El trabajo se desarrolló teniendo en cuenta 3 fases de trabajo, en las cuales se realizó la contextualización, la recolección y sistematización de la información y el análisis de resultados (Ver figura 2).

\section{FASES DE TRABAJO}

\section{FASE 1:}

Contextualización. Proyecto

Político Pedagógico (PPP),

Proyecto Curricular de Licenciatura en Biología (PCLB).

\section{FASE2:}

Recolección y sistematización de la información. Programas de semestres, documentos relacionados con las prácticas de campo y el programa del espacio académico biológico, informes de salidas y primera entrega del proyecto de semestre. Observación participante, adaptación, tutorías y plenarias Uso de instrumentos como entrevistas encuestas y cuestionarios.

\section{FASE 3:}

Análisis de resultados. Análisis de los resultados y presentación al grupo de investigación y al Departamento de Biología.

Figura 2: Fases de la Investigación

\section{CONTEXTUALIZACIÓN:}

Es necesario tener en cuenta algunos aspectos con el fin de contextualizar el grupo con el cual se desarrolló la investigación.

La Universidad Pedagógica Nacional (UPN), es una institución pública encargada de formar profesionales de la educación en diferentes disciplinas, entre las que se encuentra Licenciatura en Biología (LB). Esta Licenciatura se desarrolla al interior de la Facultad de Ciencia y Tecnología, en el Departamento de Biología, el cual plantea el proyecto Curricular de Licenciatura en Biología (PCLB).

La estructura del PCLB consta de dos ciclos, uno de fundamentación que comprende de primero a sexto semestre en el que las actividades académicas son comunes a todos los estudiantes y otro de profundización que va de séptimo a décimo semestre en el que los estudiantes optan por énfasis particulares.

En el ciclo de fundamentación cada semestre corresponde a un eje curricular, dentro de los cuáles se desarrollan la introducción al semestre (UI), componentes que son las materias ofrecidas a los estudiantes y un proyecto de investigación. Entre estos semestres se encuentra el eje curricular Interacción, en donde se desarrollan los espacios académicos denominados Adaptación y Pedagogía y Didáctica II (Ver Figura 2). Para 
este trabajo se tuvo en cuenta una población de 10 estudiantes de sexto semestre escogidos aleatoriamente, a los cuales se les realizó un seguimiento sobre sus ideas y se entrevistaron 6 maestros, uno por cada semestre del ciclo de fundamentación.

UNIVERSIDAD PEDAGÓGICA NACIONAL

FACULTAD DE CIENCIA Y TECNOLOGÍA

DEPARTAMENTO DE BIOLOGÍA

PROYECTO CURRICULAR DE LICENCIATURA EN BIOLOGÍA

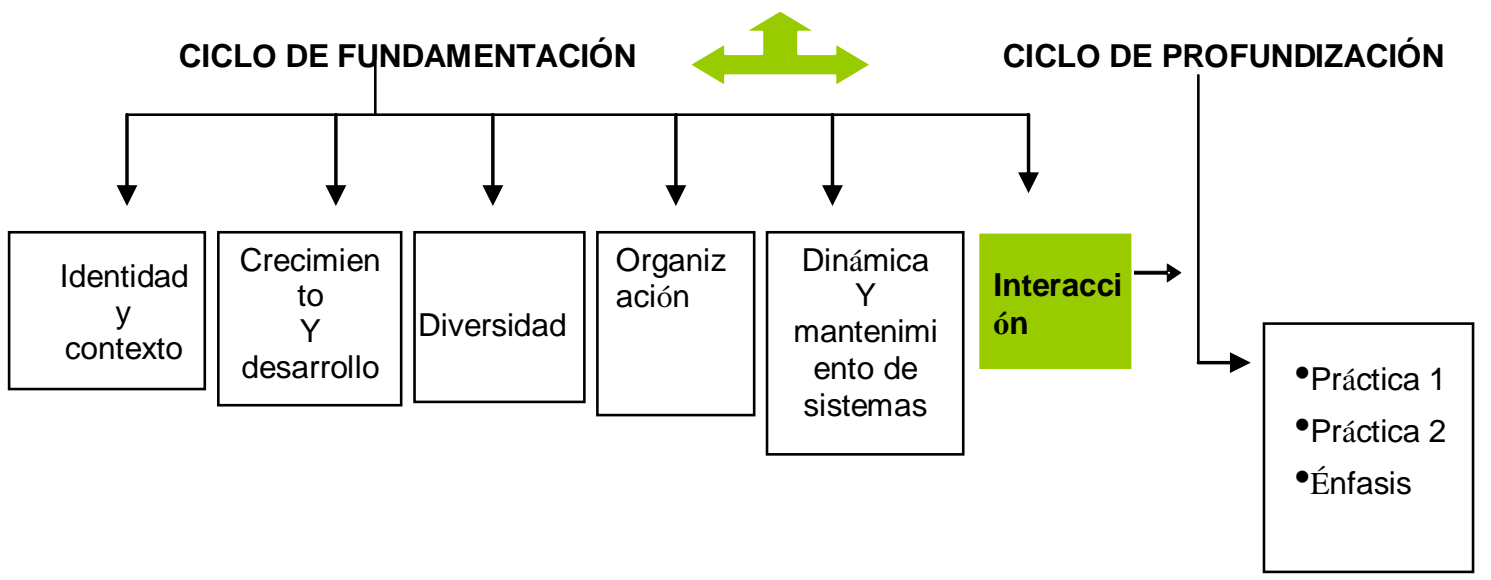

Figura 2: Contextualización del grupo de trabajo

\section{MARCO DE REFERENCIA CONCEPTUAL}

Existen dos tipos de Conocimientos que constituyen el CPPB, éstos son el Conocimiento Biológico y el Conocimiento Didáctico del Contenido Biológico. A continuación se realiza una breve descripción acerca de estos, y además su relación con los trabajos prácticos de campo.

\section{Conocimiento Biológico (CB)}

Es claro que se debe requerir un $\mathrm{CB}$ para enseñar, además se debe conocer los contenidos a enseñar, lo que permite definir objetivos, contenidos y actividades de enseñanza; también le permite al docente establecer relaciones entre los conceptos que enseña, identificar los principios fundamentales de la disciplina, identificar las aplicaciones que pueden tener los contenidos, diseñar-implementar y/o adoptar prácticas de laboratorio, transformar los contenidos de la enseñanza, detectar ideas erróneas de los alumnos y evaluar textos escolares. Este Conocimiento Biológico debe contar con un Conocimiento Histórico y Epistemológico (Valbuena 2007).

En cuanto a la producción del Conocimiento Biológico, Abrams y Wandersee proponen una secuencia del cambio en las ideas de la forma como se produce el 
Conocimiento Biológico: inicialmente con la observación directa del mundo viviente, luego a través de cómo el sujeto percibe e interactúa con el mundo viviente, luego la interacción del sujeto con el mundo viviente influenciado por las cultura, el aprendizaje y la sociedad como fuentes de información (Valbuena 2007). De esta manera se puede evidenciar que las prácticas de campo con de acuerdo a sus fines y objetivos pueden propender por la producción del CB.

\section{Conocimiento Didáctico del Contenido Biológico (CDCB):}

Haciendo referencia al otro componente del CPPB, es decir, el Conocimiento Didáctico del Contenido, Magnusson, Krajcik y Borko (1999), citado en Valbuena (2007), plantean que el profesor demanda de un Conocimiento que le permite transformar e integrar los diferentes tipos de Conocimientos, incluido el disciplinar, de esta manera identifican cinco dominios principales del CDC: las orientaciones de la enseñanza de la Ciencia, el Conocimiento del currículo de Ciencia, el Conocimiento del aprendizaje de la Ciencia por parte de los alumnos, el Conocimiento de la evaluación de los aprendizajes y el Conocimiento de estrategias metodológicas de enseñanza, ya sea de un tópico general de la Ciencia o de un tópico específico. Es en este último en donde las prácticas de campo se pueden enmarcar, además de esto, el profesor debe contar con un Conocimiento particular acerca de la metodología, las estrategias y actividades de enseñanza, lo cual implica conocer modelos, ejemplos, analogías, ilustraciones, problemas, demostraciones, simulaciones, entre otros, no desde la perspectiva instrumental sino desde la perspectiva del alcance conceptual de cada actividad.

Haciendo referencia al CDC específicamente en Biología, éste se produce a partir del proceso de transformación, procesamiento, interrelación, e integración de diversos componentes: los contenidos biológicos a enseñar, las finalidades de la Biología y de su enseñanza, las estrategias metodológicas para la enseñanza de la Biología, las concepciones de los alumnos acerca de la Biología, las dificultades en la enseñanzaaprendizaje de la Biología y la evaluación de los aprendizajes de la Biología. Es en las estrategias metodológicas para la enseñanza de la Biología, en donde se enmarcan las prácticas de campo.

Finalmente relacionando las prácticas de campo con el $\mathrm{CPPB}$, es posible integrarlos desde dos puntos de vista; el primero desde el Conocimiento Biológico, como fuente de construcción del mismo y el segundo como estrategia metodológica en la enseñanza de la Biología como componente del CDCB.

\section{Trabajos Prácticos de Campo:}

Con respecto a las prácticas de campo Del Carmen (2000) plantea, que éstas al igual que los laboratorios se enmarcan en la categoría de trabajos prácticos, que son actividades de enseñanza de las ciencias en las que los alumnos han de utilizar determinados procedimientos para resolverlas. De esta manera implican el desarrollo 
de habilidades en el uso de procedimientos científicos, motivan al estudiante, ayudan a la comprensión de los planteamientos teóricos y promueven actitudes relacionadas con el Conocimiento Científico.

De acuerdo a Brusi (1992), citado en Del Carmen y Pedrinaci (1997), el papel didáctico de las salidas es que "favorece la inmersión en el entorno, el Conocimiento del medio local, proporciona vivencias que sirven como referente para captar los cambios temporales y los ritmos en la sucesión de los fenómenos, potencia una actitud de curiosidad y ayuda a concienciar la problemática natural y social del entorno, en donde se pueden aprender ciertos procedimientos y actitudes".

Por otra parte Alarcón y Piñeros (1989), plantean que las salidas de campo son un recurso pedagógico que proporciona una interacción con la naturaleza en forma directa, fundamenta los Conocimientos, ayuda al desarrollo del individuo como persona y le permite la apropiación de los temas, enriqueciendo e interpretando las asignaturas.

Tomando posición frente a lo encontrado se considera para esta investigación, que las Prácticas de Campo se enmarcan en la categoría de trabajo práctico al igual que los trabajos de laboratorios y son una estrategia en la enseñanza de las ciencias que propician el desarrollo de habilidades y competencias en los estudiantes; y es aquí en donde se traza la discusión acerca de lo que propicia y lo que debería propiciar las salidas de campo, que debería abarcar contenidos conceptuales, procedimentales y actitudinales desde un punto de vista constructivista que permita al estudiante evolucionar sus concepciones acerca de la naturaleza y acerca del trabajo científico.

Teniendo en cuenta lo anterior, es importante ahondar sobre el trabajo práctico en la enseñanza de las ciencias, desde diversas perspectivas. El trabajo práctico constituye una referencia importante en la educación en ciencias. Tsai (2003), citado en Baldaia (2006), admite que existen diferencias significativas entre la percepción del profesorado y la percepción del alumnado sobre el trabajo práctico.

Es importante reconocer que las características del Trabajo Práctico dependen del modelo de enseñanza-aprendizaje en el que se circunscriban.

El trabajo práctico en la enseñanza por transmisión, asume un papel demostrativo. Los Conocimientos teóricos adquiridos de forma transmisiva se confirman a través de clases prácticas que motivan al estudiante. Aquí la observación tiene un papel importante en la construcción de Conocimiento. Por lo tanto, el objetivo es adquirir Conocimientos haciendo énfasis en la instrucción, en donde el docente transmite contenidos y el alumnado almacena información, lo cual debe observarse en la evaluación. Por otro lado, en la enseñanza por descubrimiento, el trabajo práctico se destina a proporcionar al alumnado la oportunidad de descubrir, desprovistos de contenidos, así el objetivo es la comprensión de los procesos científicos, en donde el docente asume el papel de organizador de las situaciones de aprendizaje, además el 
alumnado aprende siguiendo el método científico, en donde ellos son el centro del aprendizaje y su evaluación se centra en los procesos científicos.

En cuanto a la enseñanza por cambio conceptual, el trabajo práctico permite diagnosticar las concepciones del alumnado y crear oportunidades para el cambio conceptual, primero el estudiante explicita lo que ya sabe, el trabajo le crea un conflicto cognitivo y finalmente un cambio conceptual. Finalmente en la enseñanza por investigación, el trabajo práctico deberá desencadenar la vivencia de la verdadera racionalidad que opera entre la instancia lógica y la instancia empírica; partiendo de la vida cotidiana del alumnado. Es por eso que el objetivo aquí es construir conceptos, competencias y valores, en donde el docente asume el papel de problematizador de saberes y organizador de procesos de aprendizaje, cuyo papel de alumnado es activo en la investigación crítica que finalmente es evaluado integralmente en conceptos, capacidades y actitudes (Baldaia, 2006).

En cuanto al trabajo práctico y la formación del profesorado en la enseñanza de las ciencias, este ha venido constituyendo una faceta muy valorada entre los enseñantes (Boyer y Tiberghien 1989; Rodrigo 1993; García Barros 1998; citados en Rodrigo et al, 1999). Los estudios realizados sobre la efectividad de estas actividades, son escasos; también son escasos los estudios específicos de concepciones del profesorado relacionados con maneras de abordar el trabajo práctico. Para estos estudios es esencial conocer las ideas de partida del futuro profesorado y así hacer una aproximación a las concepciones que tienen sobre el trabajo práctico (Rodrigo et al, 1999).

Dado que se indagó algunas concepciones de los futuros profesores, es importante definirlas.

Las concepciones, o concepciones alternativas de los estudiantes no son explícitas, son modelos explicativos, que pueden evolucionar a medida que se construye el Conocimiento, de tal manera que hacen uso de esas concepciones de manera variable. Las concepciones tienen una génesis individual y social, en donde interviene la escuela, la familia y los medios de comunicación junto con la actividad social. Estas concepciones se caracterizan por ser respuestas rápidas, seguras sin ser sometidas a un análisis (De Posada, J 2000).

\section{RESULTADOS Y DISCUSION}

\section{INDAGACIÓN DE IDEAS}

\section{Maestros}

Como ya se mencionó, en primera medida se indagó las ideas que tenían tanto profesores como estudiantes sobre las Prácticas de Campo y su relación con el CPPB.

A continuación se muestran algunos de los datos obtenidos a partir de la entrevista realizada a los coordinadores de los primeros seis semestres. 
Con respecto a la pregunta ¿Cómo Se está aportando a la formación profesional a partir del trabajo con las prácticas de campo?, un docente respondía:

\begin{abstract}
FORMADOR DE SEGUNDO SEMESTRE: “...Este trabajo aporta de muchas maneras (...) permite conocer mejor el país (...) trabajar con salidas para integrarse (...) les aporta a cómo se colaboran en las salidas, como ayudarse, ya que ellos también harán salidas (...) lo otro es las guías y cómo se llevan, eso también aporta a la formación..."
\end{abstract}

Al entrevistar a los maestros coordinadores de cada semestre del ciclo de fundamentación acerca de cómo las Prácticas de Campo favorecían la formación de sus estudiantes, se evidenció en un referente mayoritario, que éstas pretenden aportar a la constitución del ser docente desde su formación pedagógica y disciplinar teniendo en cuenta las características de cada semestre.

\title{
Estudiantes
}

Por otra parte al aplicar un cuestionario a los futuros maestros (Ver anexo 1: Cuestionario para la indagación de concepciones del Trabajo Práctico de Campo, modificado de Rodrigo et al, 1999) algunos estudiantes futuros profesores de sexto semestre respondieron a la pregunta ¿Cómo ha contribuido el trabajo con prácticas de campo a tu formación como Licenciado en Biología? Los números corresponden a códigos de los estudiantes, de tal manera que se pudo hacer un seguimiento a sus ideas y evidenciar las respuestas mayoritarias:

- Me han permitido un mejor acercamiento a la comprensión y construcción de los temas, conceptos, etc. y relacionarlos con la teoría, aplicando habilidades (7 estudiantes E2, E3. E4, E5, E8, E9, E10)

- Me ha dado una perspectiva de cómo realizar este tipo de trabajos, de la utilidad, de las necesidades de la preparación y las actividades post. (1 estudiante E1)

Al observar los resultados tanto la entrevista como el cuestionario, se evidenció que existe una discrepancia entre lo que plantean los maestros y lo que plantean los estudiantes acerca de la contribución de las prácticas de campo a su formación docente, ya que para los estudiantes, la contribución a la formación del licenciado en Biología a partir del trabajo con salidas de campo, radica fundamentalmente en su aporte al Conocimiento Biológico, aportando a partir del encuentro con situaciones cotidianas y problémicas, el desarrollo de habilidades, conceptos, procedimientos y actitudes hacia lo biológico, de tal manera los estudiantes se acercan a la producción del Conocimiento científico y no a la integración del Conocimiento profesional a partir 
de la constitución de su CDCB; las prácticas de campo les aporta en la construcción de su Conocimiento Biológico únicamente, sin plantear que éstas constituyen parte fundamental en la enseñanza de la biología y por ende en su futuro quehacer docente.

Partiendo de la situación anterior, se propuso favorecer la apropiación de la práctica de campo como estrategia en la enseñanza de la biología en los estudiantes de sexto semestre.

\section{Diseño de la Práctica de Campo}

Para favorecer dicha apropiación se planteó un espacio al interior del espacio académico de Pedagogía y Didáctica II en donde los futuros profesores diseñaron una Práctica de Campo para alumnos de segundo grado de una institución educativa distrital; para dicho trabajo, se tuvo en cuenta tres fases, una previa, una durante y una posterior. Estas fases a partir de las propuestas de Alarcón y Piñeros (1989) y Del Carmen y Pedrinaci (1997).

\section{Fase previa}

Durante la fase previa, los futuros profesores desarrollaron diversos talleres, en donde pudieron recordar y discutir en grupos de trabajo, los objetivos, las metodologías, los lugares y demás aspectos acerca de las Prácticas de Campo que habían vivenciado durante su formación inicial, y a partir de allí plantear cómo ellos la hubieran diseñado, teniendo en cuenta su formación como profesores de biología, de tal manera que a través de este ejercicio se acercaran al diseño de una práctica de campo, teniendo en cuenta aspectos como, objetivos formativos, metodologías de trabajo, evaluación, entre otros.

Particularmente con respecto a los objetivos formativos, los estudiantes de sexto semestre explicitaron lo siguiente:

E3 y E10: [General]

- “...Reconocer el trabajo en campo como una estrategia metodológica para la enseñanza de las ciencias naturales, involucrando los intereses de maestros y estudiantes..."

[Específicos]

- "...Identificar las metodologías adecuadas para realizar un trabajo en campo..."

E4: [General] 
- "...Evidenciar la importancia del trabajo de campo en la formación docente a partir de la interacción con los estudiantes de grado..."

\section{[Específicos]}

- "...Determinar la pertinencia de las salidas de campo en la enseñanza de la biología..."

- “...Establecer una metodología de campo que permita relacionar la teoría con la práctica en la enseñanza de la biología..."

- “...Reflexionar sobre las implicaciones del ser docente en biología a partir del proceso enseñanza aprendizaje que se desarrollará en la salida de campo..."

Así mismo, los estudiantes se acercaron a la conceptualización de las Prácticas de Campo, de tal manera que revisaron diversos autores y definiciones de dicha estrategia, permitiéndoles relacionar la experiencia vivida durante su ciclo de fundamentación con lo planteado por diversos autores.

La reflexión sobre la enseñanza de la biología, estuvo también enmarcada desde el espacio académico Adaptación, en cuyas guías de campo se incluyeron preguntas como ¿Cuál crees que es el aporte de esta salida en tu formación disciplinar?, Según el trabajo realizado ¿crees que la metodología planteada por el profesor permitió el cumplimiento de los objetivos? Si pudieras diseñar una práctica de campo, ¿Qué tipo de contenidos abordarías?, ¿Cómo evaluarías los aprendizajes? Y finalmente ¿Consideras importante que en la formación inicial de los profesores, se incluya el diseño de prácticas de campo en términos disciplinares y pedagógicos? Esta serie de preguntas, permitieron a los estudiantes reflexionar sobre la enseñanza de la biología, de nuevo desde su propia formación y proceso de aprendizaje, ya que discutieron sobre el papel del profesor en las prácticas de campo, las mejores y más pertinentes metodologías de trabajo, reconociendo de tal manera la importancia de ligar la teoría con la práctica y la importancia de reconocer el entorno natural como un escenario de enseñanza-aprendizaje, que se debe favorecer desde la formación inicial del profesor de biología.

En esta fase previa, los estudiantes también diseñaron las guías de campo con las cuales realizaron la práctica de campo, teniendo en cuenta aspectos como los estándares, objetivos, actividades y las características de sus alumnos que pudieron identificar en una visita previa a la institución educativa. En la fase del durante, se desarrollo la práctica de campo con los alumnos de segundo grado. Y en la fase posterior, los futuros profesores aunque por falta de tiempo no pudieron evaluar el aprendizaje de sus alumnos, plantearon algunas sugerencias para continuar con el 
proceso académico de los alumnos en la institución educativa. Al finalizar, los futuros profesores evaluaron su propio trabajo acerca del diseño de la práctica de campo.

Con respecto a los contenidos biológicos a enseñar que plantearon los futuros profesores para la Práctica de Campo, se plantearon entre ellos en la primera aplicación del cuestionario:

- "En las salidas se trabajan contenidos nuevos, pienso que es muy importante realizar una preparación de los contenidos". E1.

Este referente corresponde a un nivel inicial de acuerdo a la propuesta de Valbuena (2007); en este nivel no se explicitan el tipo de contenidos de enseñanza, es decir que no hacen explícito qué tipo de contenidos son los que se deberían afianzar, es decir si únicamente son conceptuales, o si también se incluyen procedimentales y actitudinales.

A diferencia de lo anterior, durante el diseño de la Práctica, cuando diseñaron los objetivos para los alumnos, fue evidente que los futuros profesores tuvieron en cuenta para la salida contenidos conceptuales, procedimentales y actitudinales en torno a los seres vivos como se muestra a continuación:

- "...Reconocer la incidencia que tiene el hombre en las dinámicas de mi entorno..". E2.

- " “..Describir cómo soy y cómo son otros seres vivos..." E2

- "...Reflexiono sobre la importancia de cuidar el entorno..." E4.

- "...Reconozco y describo la flora y fauna que se encuentra en mi entorno..." E9.

\section{Fase posterior}

El diseño de la Práctica de Campo para alumnos de segundo grado de una Institución Educativa Distrital fue una actividad transversal durante todo el periodo académico, durante el cual se realizó un seguimiento al trabajo elaborado por los estudiantes, a través de instrumentos como cuestionarios, encuestas y socializaciones aplicadas a los futuros profesores.

A la pregunta ¿Qué aporta para tu formación docente el haber diseñado una práctica de campo? Planteada en uno de los cuestionarios, algunos de los estudiantes de sexto semestre respondieron:

- “...Esta clase de prácticas [De campo] fomenta actitudes positivas hacia la elaboración de este tipo de actividades, promoviendo la investigación de los estudiantes. Contribuye a que como estudiantes en formación, seamos participes en la construcción de nuestro propio conocimiento..." E2. 
- “...Reconocer que éstas prácticas son herramientas muy importantes en las ciencias naturales..." E8.

Teniendo en cuenta los instrumentos empleados, se pudo evidenciar que la actividad del diseño de la práctica de campo contribuyó a que los futuros profesores reflexionaran sobre dos aspectos muy importantes, la práctica de campo y la formación profesional; de tal manera que relacionaron la práctica de campo con la enseñanza de la biología y además plantearon que dicha relación se puede enmarcar en la formación docente, e incluso mas relevante, desde el Conocimiento Profesional del Profesor, lo cual va muy de acuerdo con lo planteado en el CDCB por Valbuena (2007).

El diseño de la práctica de campo, permitió que los profesores en formación integraran diversos Conocimientos, lo disciplinar, lo didáctico y lo pedagógico, dentro de una estrategia como la práctica de campo.

Cabe aclarar que este diseño de la práctica de campo, también les permitió a los futuros profesores de biología reflexionar sobre aspectos muy importantes en la enseñanza de la biología, tales como los objetivos educativos, y por ende contenidos conceptuales, procedimentales, actitudinales, actividades de enseñanza, indagación de ideas previas, formas de evaluar, metodologías de trabajo, entre otras.

\section{CONCLUSIONES}

Aunque en una primera instancia se había afirmado que existe diferencias entre lo que piensan los profesores y lo que piensan sus estudiantes sobre cómo las prácticas de campo contribuyen a la formación docente y profesional de los futuros profesores de biología, esta práctica pedagógica permitió que en cierta medida los planteamientos de maestros y estudiantes tuvieran puntos de encuentro.

Las ideas de los futuros profesores pudieron progresar a través de las actividades que se desarrollaron en el semestre, tales como talleres, guías y el diseño de una práctica de campo, sobre todo en dos aspectos como lo son la relación entre las prácticas de campo y el CPPB, los objetivos de aprendizaje y los contenidos a enseñar.

Es muy importante por lo tanto favorecer procesos de reflexión que permitan a los futuros profesores de biología construir su Conocimiento Didáctico del Contenido Biológico, a partir de la apropiación de las prácticas de campo como una estrategia en la enseñanza de la biología. Dicha reflexión se puede enmarcar dentro de los componentes pedagógicos o didácticos y disciplinares biológicos de los programas de formación de profesores, para este caso el componente de de pedagogía y didáctica 2, y también a partir del componente de adaptación, permitiendo a los estudiantes reconocer que su experiencia es fundamental en su futuro quehacer docente. Claro está 
que dicha reflexión podría ser transversal por lo menos durante el ciclo de fundamentación a partir del ambiente de pedagogía en estos semestres.

Al interior del Proyecto Curricular de Licenciatura en Biología, es muy relevante crear espacios en los que los futuros profesores se acerquen al diseño de prácticas de campo, que les permita favorecer sus procesos de aprendizaje en términos de la enseñanza de la biología, en el marco del Conocimiento Profesional del Profesor de Biología; actividades como los talleres, guías y el planteamiento de preguntas en las guías de campo favorecen la reflexión sobre dicho Conocimiento, que pueden confluir positivamente en el diseño de una práctica de campo. De igual forma, estos planteamientos se pueden hacer extensivos para cualquier programa de formación inicial de profesores de biología en diversos contextos, incluso se debería tener en cuenta en general en la formación del profesorado en ciencias.

\section{BIBLIOGRAFÍA}

- Alarcón, \& Piñeros, I. (1989). Las salidas de campo como un recurso pedagógico. Modelo de una salida. Trabajo de grado de licenciado en biología y química. Universidad de la Salle. Facultad de ciencias de la educación. Bogotá. 106 p.

- Baldaia, L. (2006). El cambio de las concepciones didácticas sobre las prácticas, en la enseñanza de la biología. Revista Alambique: Didáctica de las ciencias experimentales. 47 (1): 23-29.

- Bonilla, E y Rodríguez, P. (1997). Más allá del dilema de los métodos. Grupo editorial Norma. Santa Fe de Bogotá. 220p.

- Del Carmen, L. (2000). Los trabajos prácticos. p 267-287. En Perales, F y Cañal, P (eds.). Didáctica de las ciencias experimentales. Editorial Marfil. Alcoy. España. 703p.

- Del Carmen, L y Pedrinaci, E. (1997). El uso del entorno y el trabajo de campo. p 133-154. En: Del Carmen, L (coordinador). La enseñanza y el aprendizaje de las ciencias de la naturaleza en la educación secundaria. Editorial Horsori. Barcelona. España. 222p.

- De Posada, J.(2000). El estudio didáctico de las ideas previas. p 363-388. En: Perales, F y Cañal, P (eds.). Didáctica de las ciencias experimentales. Editorial Marfil. Alcoy. España. 703p.

- Rodrigo M; Morcillo G; Borges R; Calvo M; Cordeiro N; García F; Raviolo A. (1999). Concepciones sobre el trabajo práctico de campo (TPc); una aproximación al pensamiento de los futuros profesores. En: Revista Complutense de Educación. 10 (2), 261-285.

- Miles y Huberman. (1994). Qualitative data analysis: an expanded sourcebook. Thousands Oaks. 338p.

- Valbuena, E. (2007). El Conocimiento Didáctico del Contenido Biológico. Estudio de las concepciones disciplinares y didácticas de futuros docentes de la Universidad Pedagógica Nacional (Colombia). Tesis para optar al título de Doctor en Didáctica de las Ciencias Experimentales. Universidad Complutense de Madrid. Madrid. 633p. 


\section{ANEXOS}

ANEXO1: CUESTIONARIO PARA LA INDAGACIÓN DE CONCEPCIONES
ACERCA DEL TRABAJO PRÁCTICO DE CAMPO

UNIVERSIDAD PEDAGÓGICA NACIONAL

FACULTAD DE CIENCIA Y TECNOLOGÍA

DEPARTAMENTO DE BIOLOGÍA

PRÁCTICA PEDAGÓGICA 1

Elías Amórtegui

2004210003

Practicante del DBI

El presente cuestionario se plantea con el fin de hacer una aproximación a las concepciones que tienen los estudiantes de sexto semestre, acerca de las prácticas de campo y su contribución a la formación de licenciados en Biología.

\section{Objetivos formativos para los docentes}

Asumiéndote como docente, ¿las prácticas de campo con tus estudiantes, serían esenciales en la enseñanza de la biología? Argumenta tu respuesta.

\section{Modelo de Enseñanza}

A continuación se describen cuatro metodologías para trabajar las salidas de campo.

¿Cuál de estas metodologías te parece mejor para trabajar las salidas de campo durante el ciclo de fundamentación? (Primera columna) Y ¿Cuál es, según tu experiencia, la más frecuente en el Proyecto Curricular de Licenciatura en Biología? (Segunda columna)

\begin{tabular}{|l|l|}
\hline \multicolumn{1}{|c|}{ Mejor } & \multicolumn{1}{c|}{ Más frecuente } \\
\hline En primer lugar la & $\begin{array}{l}\text { En primer lugar la } \\
\text { № }\end{array}$ \\
& \\
\hline
\end{tabular}


1. Seleccionar los mejores lugares y

\begin{tabular}{|l|l|}
\hline $\begin{array}{l}\text { En segundo lugar } \\
\text { la № }\end{array}$ & $\begin{array}{l}\text { En segundo lugar } \\
\text { la № }\end{array}$ \\
& \\
\hline
\end{tabular}
interesantes. Los alumnos observan, toman notas, realizan esquemas etc.

2. Seleccionar los mejores lugares y allí formular preguntas intentando que los alumnos resuelvan problemas puntuales.

3. Los alumnos trabajan realizando actividades guiadas secuencialmente por un guión o guía didáctica.

4. Los alumnos realizan una investigación con bastante autonomía. El papel del profesor es el de tutor.

Argumenta tu respuesta

- En tu opinión, durante las salidas de campo, es preferible que los alumnos trabajen: (Marca con una X)

\begin{tabular}{|l|l|l|}
\hline Individualmente & En grupos de alumnos & Todos juntos \\
\hline & & \\
\hline
\end{tabular}

Argumenta tu respuesta:

\section{Relaciones entre conocimientos teóricos y trabajos prácticos}

¿Cuándo es mejor programar las prácticas de campo? (Primera columna), y ¿Cuál ha sido la programación más frecuente en tu formación en el ciclo de fundamentación? (Segunda columna).

1. Una vez estudiada la teoría. Al final del tema.

2. Antes de abordar un tema, como paso inicial del mismo.

3. En conjunción con la teoría, de tal forma que teoría y práctica constituyen partes integradas de los componentes.

\begin{tabular}{|c|c|}
\hline Mejor & Más frecuente \\
\hline $\begin{array}{l}\text { En primer } \\
\text { lugar la № }\end{array}$ & $\begin{array}{l}\text { En primer } \\
\text { lugar la № }\end{array}$ \\
\hline
\end{tabular}

Argumenta tu respuesta 


\section{Preparación del trabajo práctico}

¿Cuál de estos cuatro tipos de preparación sobre contenidos es, en tu opinión más conveniente para las salidas de campo?

1. La salida es al final, como repaso o enriquecimiento del curso o de parte de él. No es necesaria la preparación sobre los contenidos.

2. La salida es al final, como repaso o enriquecimiento del curso o de parte de él. Es conveniente una preparación para repasar contenidos ya trabajados.

3. En la salida trabajamos contenidos nuevos. No es necesaria una preparación sobre los contenidos, ya que los trabajamos solamente durante la salida.

4. En la salida trabajamos contenidos nuevos. En las clases anteriores es conveniente realizar una preparación específica para ella.

Argumenta tu respuesta

Concretar el tipo de preparación que considera más adecuado:

A continuación se describen cuatro preparaciones para las salidas de campo.

¿Cuál/es consideras necesaria? (Primera columna SI) ¿Cuál/es consideras que NO es necesaria? (Segunda columna NO)

1. Preparación sobre los contenidos; basada en actividades concretas sobre los conceptos y destrezas que se van a necesitar en la salida.

2. Preparación sobre la Geografía; preparar bien la localización y rasgos de la zona a visitar mediante mapas, perfiles, diapositivas o video.

3. Preparación psicológica; describir con detalle el recorrido, su longitud y duración, las características

\begin{tabular}{|l|}
\hline \begin{tabular}{l} 
Mas \\
Conveniente \\
\hline El № \\
\\
\end{tabular} \\
\hline
\end{tabular}
del trabajo y los requisitos de aprendizaje en cada parada, el lugar y duración de los descansos, etc. 
4. Antes de cada salida encargar a los alumnos un trabajo previo sobre la zona a visitar.

Argumenta tu respuesta

\section{Evaluación de los alumnos}

1. ¿Piensas que el trabajo en el aula, posterior a la salida, es fundamental?

SI

$\mathrm{NO}$

2. ¿En qué debe consistir básicamente ese trabajo?

3. ¿Cómo evaluarías una salida de campo?

\section{Contribución:}

1. ¿Cómo ha contribuido el trabajo con prácticas de campo a tu formación como Licenciado en Biología? 